\title{
Correction to: Toxicity of multi-wall carbon nanotubes inhalation on the brain of rats
}

\author{
Fatemeh Samiei ${ }^{1}$. Farshad Hosseini Shirazi ${ }^{1,2}$ • Parvaneh Naserzadeh ${ }^{1} \cdot$ Faezeh Dousti $^{1}$ • Enayatollah Seydi ${ }^{3,4}$. \\ Jalal Pourahmad ${ }^{1}$
}

Published online: 16 June 2020

(C) Springer-Verlag GmbH Germany, part of Springer Nature 2020

Correction to: Environmental Science and Pollution Research (2020) 27:12096-12111 https://doi.org/10.1007/s11356-020-07740-5

Funding information This research was financially supported by National Institute for Medical Research Development (NIMAD).

Publisher's note Springer Nature remains neutral with regard to jurisdictional claims in published maps and institutional affiliations.

The online version of the original article can be found at https://doi.org/ $10.1007 / \mathrm{s} 11356-020-07740-5$

Enayatollah Seydi

enayat.seydi@yahoo.com

$\bowtie$ Jalal Pourahmad

j.pourahmadjaktaji@utoronto.ca

1 Department of Pharmacology and Toxicology, Faculty of Pharmacy, Shahid Beheshti University of Medical Sciences, P.O. Box:

14155-6153, Tehran, Iran

2 Pharmaceutical Sciences Research Center, Shahid Beheshti University of Medical Sciences, Tehran, Iran

3 Department of Occupational Health and Safety Engineering, School of Health, Alborz University of Medical Sciences, Karaj, Iran

4 Research Center for Health, Safety and Environment, Alborz University of Medical Sciences, Karaj, Iran 